\title{
ID1 mediates resistance to osimertinib in EGFR T790M-positive non-small cell lung cancer through epithelial-mesenchymal transition
}

Kejun Liu ${ }^{1 \dagger}$, Xianwen Chen ${ }^{2 \dagger}$, Ligang Wu ${ }^{1 \dagger}$, Shiyuan Chen ${ }^{1}$, Nianxin Fang ${ }^{3 *}$, Limin Cai ${ }^{1 *}$ and Jun Jia ${ }^{{ }^{*}}$

\begin{abstract}
Background: ID1 is associated with resistance to the first generation of EGFR tyrosine kinase inhibitors (EGFR-TKIs) in non-small cell lung cancer (NSCLC). However, the effect of ID1 expression on osimertinib resistance in EGFR T790Mpositive NSCLC is not clear.
\end{abstract}

Methods: We established a drug-resistant cell line, H1975/OR, from the osimertinib-sensitive cell line H1975. Alterations in ID1 protein expression and Epithelial-mesenchymal transition (EMT)-related proteins were detected with western blot analysis. RT-PCR was used to evaluate the differences of gene mRNA levels. ID1 silencing and overexpression were used to investigate the effects of related gene on osimertinib resistance. Cell Counting Kit-8 (CCK8) was used to assess the proliferation rate in cells with altered of ID1 expression. Transwell assay was used to evaluate the invasion ability of different cells. The effects on the cell cycle and apoptosis were also compared using flow cytometry.

Results: In our study, we found that in osimertinib-resistant NSCLC cells, the expression level of the EMT-related protein E-cadherin was lower than that of sensitive cells, while the expression level of ID1 and vimentin were higher than those of sensitive cells. ID1 expression levels was closely related to E-cadherin and vimentin in both osimertinibsensitive and resistant cells. Alteration of ID1 expression in H1975/OR cells could change the expression of E-cadherin. Downregulating ID1 expression in H1975/OR cells could inhibit cell proliferation, reduce cell invasion, promote cell apoptosis and arrested the cell cycle in the G1/G0 stage phase. Our study suggests that ID1 may induce EMT in EGFR T790M-positive NSCLC, which mediates drug resistance of osimertinib.

Conclusions: Our study revealed the mechanism of ID1 mediated resistance to osimertinib in EGFRT790M-positive NSCLC through EMT, which may provide new ideas and methods for the treatment of EGFR mutated NSCLC after osimertinib resistance.

\footnotetext{
*Correspondence: 24588883@qq.com; dgcailimin@163.com; jiwen6465@126.

com

${ }^{\dagger}$ Kejun Liu, Xianwen Chen and Ligang Wu contributed equally to this

work.

${ }^{1}$ Dongguan Institute of Clinical Cancer Research, Affiliated Dongguan

People's Hospital, Southern Medical University, Dongguan, China

${ }^{3}$ Dongguan Institute of Respiratory and Critical Care Medicine, Affiliated

Dongguan People's Hospital, Southern Medical University, Dongguan,

China

Full list of author information is available at the end of the article
}

(c) The Author(s) 2021. Open Access This article is licensed under a Creative Commons Attribution 4.0 International License, which permits use, sharing, adaptation, distribution and reproduction in any medium or format, as long as you give appropriate credit to the original author(s) and the source, provide a link to the Creative Commons licence, and indicate if changes were made. The images or other third party material in this article are included in the article's Creative Commons licence, unless indicated otherwise in a credit line to the material. If material is not included in the article's Creative Commons licence and your intended use is not permitted by statutory regulation or exceeds the permitted use, you will need to obtain permission directly from the copyright holder. To view a copy of this licence, visit http://creativecommons.org/licenses/by/4.0/. The Creative Commons Public Domain Dedication waiver (http://creativeco mmons.org/publicdomain/zero/1.0/) applies to the data made available in this article, unless otherwise stated in a credit line to the data. 
Keyword: ID1, Osimertinib, Drug resistance, EGFR T790M, Non-small-cell lung cancer (NSCLC), Epithelialmesenchymal transition (EMT)

\section{Background}

Lung cancer, one of the most common malignant tumors and also the primary cause of death in cancer patients worldwide, is classified into small-cell lung cancer and non-small cell lung cancer (NSCLC); the latter of which accounts for appropriately $80 \%$ of all cases of lung cancer cases [1,2]. For patients with advanced NSCLC harboring epidermal growth factor receptor (EGFR) gene mutations, which accounts for $30-50 \%$ of NSCLC cases in East Asia, the first generation of EGFR tyrosine kinase inhibitors (EGFR-TKIs) such as gefitinib and erlotinib have superior therapeutic efficacy compared to traditional platinum-based chemotherapy $[3,4]$ and the median progression-free survival (PFS) is 9-13 months. However, these patients will eventually suffered secondary drug resistance during the targeted therapy and most of them may harbor the T790M mutation in exon 20 of the EGFR gene [5]. For patients with the EGFR T790M mutation, osimertinib still shows favorable therapeutic effect [6]. The median PFS of patients with NSCLC after failure of the first generation of EGFR-TKIs treatment is significantly higher than patients receiving standard platinum-based chemotherapy [7]. However, osimertinib may also develop drug resistance.

Epithelial-mesenchymal transition (EMT) may be one of the mechanisms that mediates osimertinib resistance. Previous studies have shown that resistance to gemcitabine in EMT producing pancreatic cancer cell lines was significantly increased [8]. Similarly, EMT has been observed in fluorouracil resistant colon cancer cell lines [9], tamoxifen resistant breast cancer cell lines [10], cisplatin resistant cervical cancer cell lines [11], and gefitinib resistant lung cancer cell lines [12]. Rastogi I et al. reported that in EGFR-TKIs resistant cell lines, ZEB1 was overexpressed and E-cadherin expression was inhibited, thus inducing EMT in lung cancer cells [13]. After down-regulating ZEB1 expression with miR-200a or $\beta$-catenin siRNA, EGFR-TKIs sensitivity could be restored. Recently, it has been found that the Hedgehog signaling pathway is abnormally activated in EGFR-TKIs resistant cells [14]. Blocking the Hedgehog signaling pathway with SANT-1 could restore the expression of E-cadherin and increase the sensitivity of cells to EGFR-TKIs. In EGFR-TKIs sensitive cells, upregulation of the Hedgehog signaling pathway could inhibit the expression of E-cadherin and increase the expression of Snail and ABCG2, leading to drug resistance. Therefore, these studies suggest that EMT plays an important role in mediating EGFR-TKIs resistance in lung cancer.

The process of EMT is very complex, and involves multiple signaling pathways. EMT can be induced by the NF- $\mathrm{kB} /$ snail/PTEN loop, Notch- 2 and MAPK/mitochondria. It has been shown that when EMT occurred in tumor cells, epithelial markers such as E-cadherin were lowly expressed while mesothelial markers such as vimentin and $\mathrm{N}$-cadherin were highly expressed [15]. Therefore, inhibition of E-cadherin expression can induce EMT. The exogenous signal that promote tumor growth can inhibit the expression of E-cadherin and mediate EMT by forming complex with transcription factors such as Snail, Slug, ZEB1, SIP1 and Twist and binding to the promoter of the $\mathrm{CDH} 1$ gene [5]. In addition, many microRNAs can regulate the expression of E-cadherin and induce the production of EMT in tumor cells by regulating the transcription repressors ZEB1 and SIP1.

ID1 belongs to the basic helix-loop-helix (bHLH) transcription factor family, and is hardly expressed in adult tissues and cells [16]. However, it has been reported that ID1 is highly expressed in a variety of tumors and is involved in cell proliferation, cell cycle progression, differentiation inhibition and genomic instability. Studies have shown that ID1 can promote the growth of esophageal cancer cells through PI3K/ Akt/NF-kB signaling pathway [17]. Inhibition of ID1 can lead to the inactivation of NF- $\mathrm{kB}$, thus promoting the apoptosis of colorectal cancer cells [18]. Previous studies have also reported that ID1 is highly expressed in lung cancer cells. ID1 is associated with EGFR-TKIs resistance in lung cancer. The higher the expression of ID1, the stronger the resistance of cells to EGFR-TKIs [19]. Furthermore, the higher the expression of ID1, the worse the prognosis of NSCLC. It is suggested that ID1 may inhibit the PI3K/AKT signaling pathway through negative feedback. As NF- $\mathrm{kB}$ is the downstream signal pathway of PI3K/Akt, the increase of ID1 expression may lead to EMT through the NF- $\mathrm{kB} /$ Snail/PTEN loop as illustrated above.

The aim of our study was to reveal the mechanism of ID1 mediated resistance to osimertinib in EGFR T790M-positive NSCLC through EMT, and to provide new ideas and methods for the clinical treatment of EGFR mutated NSCLC after osimertinib resistance. 


\section{Methods}

\section{Chemicals and reagents}

Osimertinib was obtained from Selleck Chemicals (Houston, TX, USA). RPMI-1640 was product of HyClone (Logan, UT, USA) and Dulbecco's modified Eagle's medium (DMEM) and fetal bovine serum (FBS) were products of Gibco BRL (Grand Island, NY, USA). Trizol was purchased from Takara Biomedical Technology (Shiga, Japan). Cell Counting Kit-8 (CCK-8) was obtained from Dojindo (Kumamoto, Japan). Monoclonal antibodies against ID1 were products of Santa Cruz Biotechnology (Santa Cruz, CA, USA). Glyceraldehyde-3-phosphate de hydrogenase (GAPDH), E-cadherin and vimentin antibodies were purchased from Abcam (Cambridge, MA, USA).

\section{Cell culture and transfection}

The EGFR mutant human lung adenocarcinoma cell lines, H1975, were purchased from the Type Culture Collection of the Chinese Academy of Sciences, Shanghai, China. H1975/OR was obtained by gradually increasing the concentration of osimertinib into H1975 cell culture medium. Cells were cultured in RPMI-1640 medium supplemented with $10 \%$ FBS (Gibco, Grand Island, NY, USA) at $37{ }^{\circ} \mathrm{C}$ in a humidified incubator with $5 \% \mathrm{CO} 2$. All cells were grown in culture medium that is free of drugs for more than 14 days before assay. Cells were seeded into 96-well Falcon plates with $1.0 \times 10^{5}$ cells per well. H1975/ OR cells were divided into 6 groups: the untreated group, empty vector group, interference control group, interference group, overexpression group, both interference and overexpression group. Transient transfections of H1975/ OR cells were performed with the lipofectamine 2000 according to the manufacturer's instructions when the cell confluence was approximately $90 \%$. Cells were cultivated for further $48 \mathrm{~h}$ prior to the following experiments.

\section{Cell proliferation assay}

Cells were seeded at a density of $1.0 \times 10^{4}$ cells/well in a 96-well plate for 48 and $72 \mathrm{~h}$. The CCK-8 solution was added to each well prior to the endpoint of incubation. The CCK- 8 reagent was added into each well at a 1:10 (v/v) dilution per $100 \mu \mathrm{L}$ and incubated for $2 \mathrm{~h}$ at $37^{\circ} \mathrm{C}$. We quantified the results spectrophotometrically at a wavelength of $450 \mathrm{~nm}$.

\section{Transwell assay}

At $48 \mathrm{~h}$ post-transfection, cells $\left(5 \times 10^{4}\right)$ were cultured in medium without serum. A Transwell assay (pore size, $8 \mu \mathrm{m}$; Corning, Inc.) was performed to detect cell invasion. Cells $\left(2 \times 10^{5}\right.$ cells $\left./ \mathrm{ml}\right)$ were plated into the upper chamber with medium containing $1 \% \mathrm{FBS}$, which was pre-coated with Matrigel ${ }^{\circledR}$ at $37{ }^{\circ} \mathrm{C}$ for $30 \mathrm{~min}$. Medium supplemented with $10 \%$ FBS $(500 \mu \mathrm{l})$ was plated in the lower chambers. Following incubation for $48 \mathrm{~h}$ at $37{ }^{\circ} \mathrm{C}$, cells on the upper chamber surface were removed. Invading cells were fixed with $50 \%$ methanol for $30 \mathrm{~min}$ at $4{ }^{\circ} \mathrm{C}$ and stained with $0.1 \%$ crystal violet for $30 \mathrm{~min}$ at room temperature. Stained cells were visualized under a light microscope and the number of invading cells was calculated to determine the relative invasion rate.

\section{Western blot analysis}

Cells were lysed after washing 2 times with ice-cold phosphate-buffered saline (PBS). The protein concentration was quantified using the Bradford method. Equal amounts of protein were resolved by sodium dodecyl sulfate polyacrylamide gel electrophoresis (SDS-PAGE) and transferred onto nitrocellulose membranes. Membranes were blocked with $5 \%$ fat-free milk combined with Trisbuffered saline for one hour at room temperature and then incubated with the appropriate primary antibody and horseradish peroxidase conjugated secondary antibody. Chemiluminescence was used to detect the proteins. Protein expression was quantified using Image-Pro Plus software (version 6.0; Media Cybernetics, Inc.) with GAPDH as the loading control.

\section{Reverse transcription PCR}

ID1 expression was assayed as described. Total RNA was isolated using the Trizol reagent RNA extraction kit (Takara Bio Inc., Shiga, Japan) according to the manufacturer's protocol. Then, cDNA was synthesized using the BestarTM qPCR RT kit (DBI Bioscience), following the manufacturer's instructions. Quantitative realtime PCR analysis was performed with the BestarTM qPCR MasterMix (DBI Bioscience). Each sample was run in triplicate for each gene. Transcript levels were normalized to the housekeeping gene phosphoglycerate kinase (PGK) and analyzed by the relative quantification $2-\Delta \Delta \mathrm{Ct}$ method. The PCR primers were as follows: GAPDH: forward: 5'-TGTTCGTCATGGGTGTGA AC-3', reverse:5'-ATGGCATGGACTGTGGTCAT-3', Vimentin:forward:5'-AGTCCACTGAGTACCGGA GAC-3', reverse:5'-CATTTCACGCATCTGGCGTTC3', E-cadherin: forward:5'-ATTTTTCCCTCGACACCC GAT-3', reverse:5'-TCCCAGGCGTAGACCAAGA-3', ID1:forward:5'-CTGCTCTACGACATGAACGG-3', reverse:5'-GAAGGTCCCTGATGTAGTCGAT-3'. The products were resolved using gel electrophoresis $(1.5 \%$ agarose gel).

\section{Flow cytometry analysis of apoptosis}

Cells were incubated with $0.25 \%$ trypsin. Then, the cells were observed under the microscope. When the cytoplasm was retracted and the cells were detached, the 
trypsin was removed. Then, $4 \mathrm{ml}$ of complete culture medium was added to stop digestion and single cell suspension was made. The single cell suspensions were transferred to flow tubes, washed with PBS twice and centrifuged at $1000 \mathrm{rpm}$ for $5 \mathrm{~min}$. The supernatants were discarded, then $200 \mu \mathrm{l}$ annexin V-FITC/propidium iodide (PI) staining solution was used to resuspend the cells. The cells were incubated in the dark for $15 \mathrm{~min}$ and staining was detected using a flow cytometer.

\section{Flow cytometry analysis of the cell cycle}

To determine the effects of ID1 on the cell cycle, $2 \times 10^{4}$ cells/well were seeded in 6-well plates and incubated for $24 \mathrm{~h}$. Cells were removed and single cell suspensions were made. The single cell suspensions were then transferred to flow tubes, washed with PBS twice and centrifuged at $1000 \mathrm{rpm}$ for $5 \mathrm{~min}$. The supernatant was completely removed as much as possible, and $1 \mathrm{ml} \mathrm{0.25 \%}$ trypsin was added. Cells were resuspended with $70 \%$ ethanol precooled at $-20{ }^{\circ} \mathrm{C}$. The samples were stored at $-20{ }^{\circ} \mathrm{C}$. The samples were centrifuged at $1000 \mathrm{rpm}$ for 5 min each time, washed with PBS for 1-2 times and centrifuged again at $1000 \mathrm{rpm}$ for $5 \mathrm{~min}$. Next, $300 \mu \mathrm{l}$ annexin V-FITC/PI staining solution was added to cells and incubated for $15 \mathrm{~min}$ in the dark, then the staining was detected and analyzed using a flow cytometer.

\section{Statistical analysis}

Statistical analyses were performed using SPSS software (version 22.0; IBM Corp). Data are presented as the mean \pm standard deviation. All experiments were repeated at least three times and the differences were analyzed by using the Student's t-test. The significance was determined at $\mathrm{P}<0.05$.

\section{Results}

Generation of the osimertinib-resistant cell line H1975/OR

We selected the NSCLC cell line H1975 in our study. H1975 cells harbor the EGFR T790M mutation and are therefore sensitive to osimertinib. Its corresponding drug resistant cell line H1975/OR was induced by osimertinib. In detail, H1975 cells were exposed to the culture medium with increasing concentration of osimertinib. Once the resistant cell line was confirmed, H1975/ OR cells were screened by gene sequencing. We found that there were no secondary gene mutations occurred, such as C797S, amplifification of MET or loss of EGFR T790M. These cells were cultured in RPMI-1640 medium containing $10 \% \mathrm{FBS}$ at $37{ }^{\circ} \mathrm{C}$ with $5 \% \mathrm{CO}_{2}$.

\section{Different expression of ID1 and EMT related protein}

In the study, we detected the expressions of ID1 and EMT-related protein at the mRNA and protein level using reverse transcription-PCR (RT-PCR) and western blot analysis. At the protein level, ID1 and vimentin appeared to be up-regulated in the H1975/OR cell line as compared to the osimertinib-sensitive cell line H1975, whereas the expression of E-cadherin was significantly decreased in H1975/OR cells (Fig. 1 and Additional files 1 and 2). The mRNA levels of ID1, vimentin and E-cadherin were also changed accordingly. These results suggested that ID1 expression level was closely related to

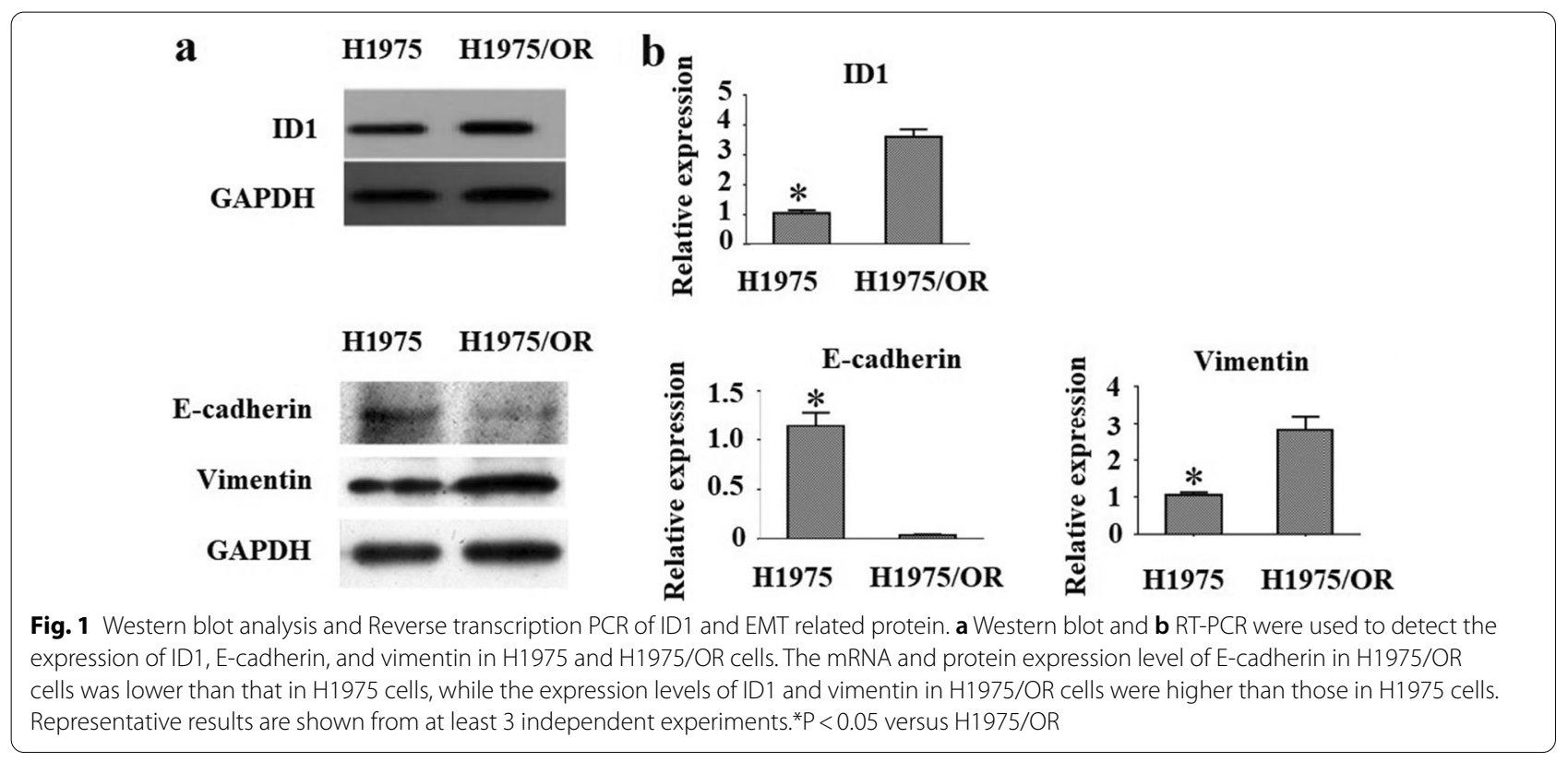


E-cadherin and vimentin both in both osimertinib-sensitive and resistant cells. Therefore, overexpression of ID1 may be one of the mechanisms of osimertinib resistance to EGFR T790M-positive NSCLC cells through EMT.

\section{Impact of ID1 on the expression of EMT-related proteins}

To further understand and investigate the overexpression of ID1 in relation to osimertinib resistance in H1957/OR cells, we performed gene silencing using siRNA targeting ID1 and overexpression by cloning the ID1 gene into the pcDNA3.0 vector. RT-PCR and western blot confirmed the effect of ID1 silencing and overexpression on drug-resistant cells. Down-regulation of ID1 expression in H1975/OR cells promoted the expression of E-cadherin and decreased the expression of vimentin (Fig. 2 and Additional file 3). Conversely, in the negative control cell line, both the expression of E-cadherin and vimentin did not changed significantly as compared to that in the H1957/OR cell line. Our study indicated that overexpression of ID1 could induce EMT in EGFR T790M-positive NSCLC cells, which may mediate drug resistance to osimertinib.

\section{Impact of ID1 on the proliferation and invasion} of osimertinib-sensitive and resistant cells

We then investigate whether alteration in ID1 expression could change cell proliferation of H1975/OR cells. As is shown in Figs. 3 and 4, we observed that downregulating ID1 could inhibit cell proliferation and reduce cell invasion, while overexpression of ID1 could further increase cell proliferation and enhance cell invasion. In control cells, the cell biological behaviors did not change significantly as compared to that of H1975/OR cells.
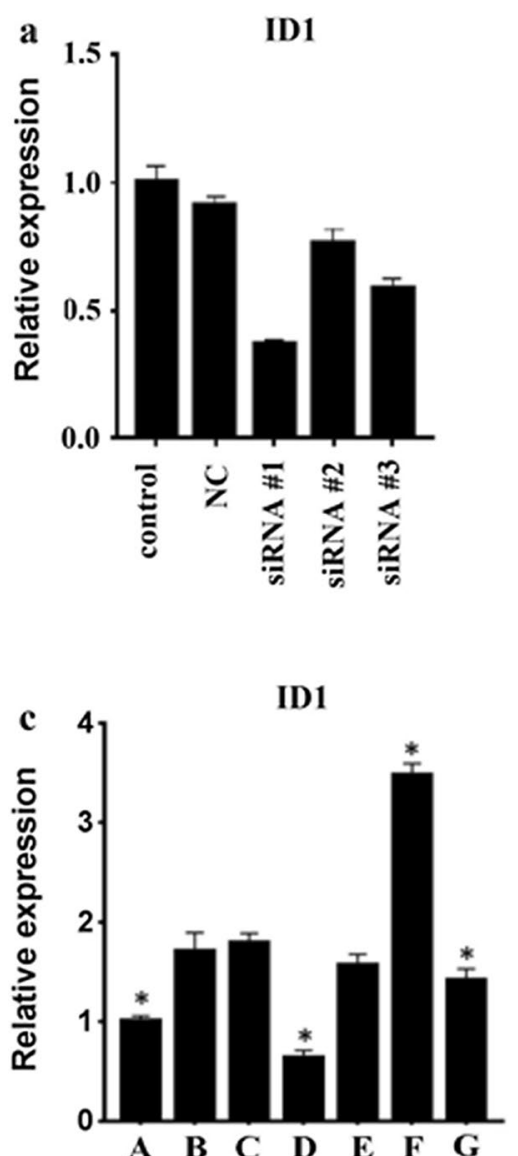

b

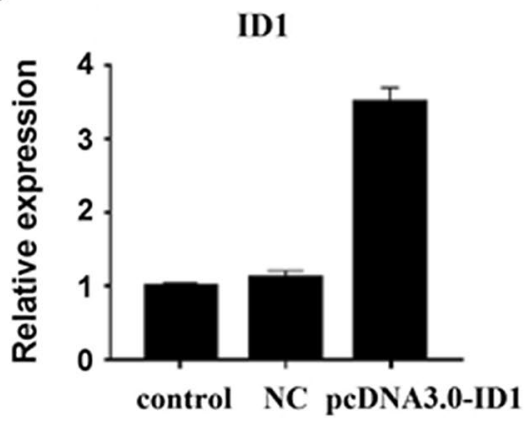

A: $\mathrm{H} 1975$

B: $\mathrm{H} 1975 / \mathrm{OR}$

C: H1975/OR+si-NC

D: H1975/OR+si-ID1

E: H1975/OR+pcDNA3.0

F: H1975/OR+pcDNA3.0-ID1

G: H1975/OR+si-ID1+pcDNA3.0-ID1

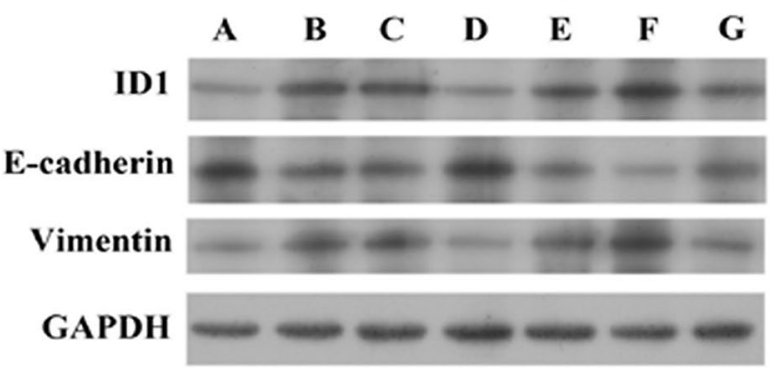

Fig. 2 Ralationship between different ID1 expression and EMT related protein. a Gene silencing using siRNA targeting IDI and $\mathbf{b}$ overexpression of ID1 by cloning the gene into the pCDNA3.0 vector. $\mathbf{c}$ and $\mathbf{d}$ RT-PCR and western blot were used to confirmed the effects of ID1 silencing and overexpression on osimertinib-resistant cells. Results showed that the expression of ID1 was closely related to E-cadherin and vimentin. Up- and down-regulation of ID1 expression in H1975/OR cells inhibited and promoted the expression of E-cadherin, respectively. Each point represents the mean \pm SD from 3 independent experiments. ${ }^{*} P<0.05$ versus $H 1975 / O R$ 


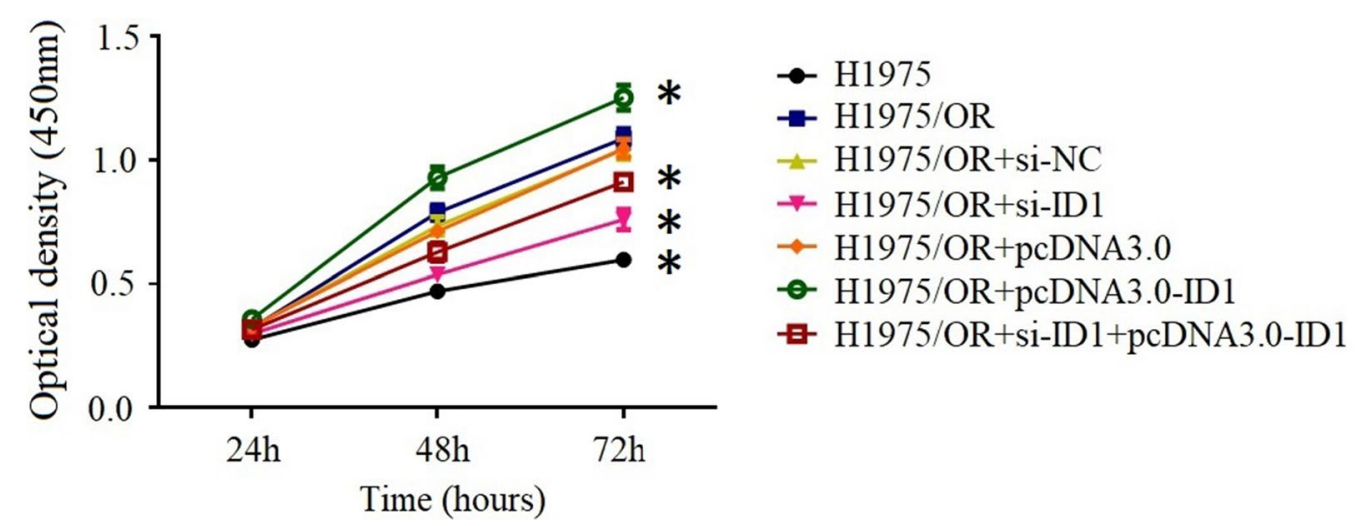

Fig. 3 Cell proliferation assay of osimertinib-sensitive and resistant cells. Results showed that cell proliferation rate was lowest in H1975 cells. Down-regulating ID1 could decrease proliferation rate in H1975/OR + si-ID1 cells, while overexpression of ID1 could further increase cell proliferation in H1975/OR + pcDNA3.0-ID1 cells. The values represent mean \pm SD from 3 independent experiments. ${ }^{*} \mathrm{P}<0.05$ versus H1975/OR

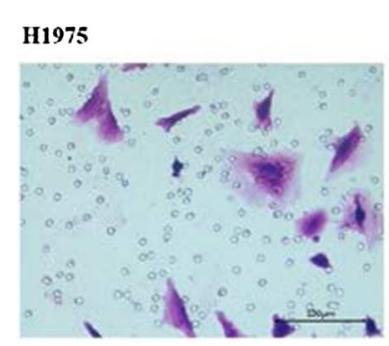

H1975/OR

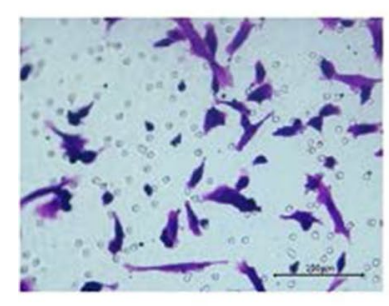

H1975/OR+pcLNA3.0

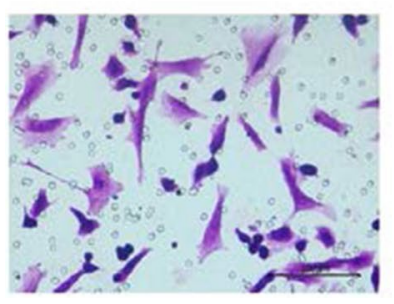

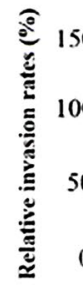

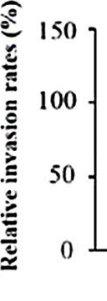

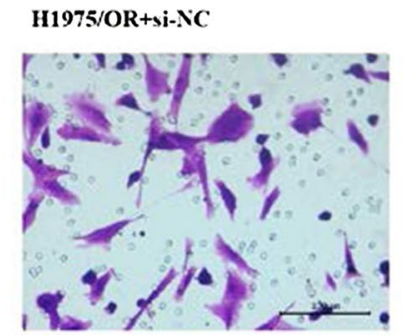

II1975/OR+pclDNA3.0-II)1

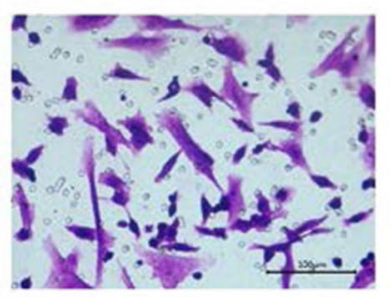

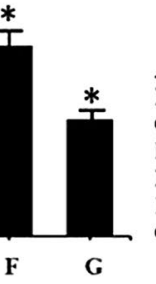

A: $\mathrm{H} 1975$

B: H1975/OR

C: H1975/OR+si-NC

D: $H 1975 / O R+s i-I D 1$

E: $\mathrm{H} 1975 / \mathrm{OR}+$ pcDNA3.0

F: H1975/OR+pcl)NA3.0-IID1

G: H1975/OR+si-ID1+pcDNA3.0-ID1

\section{H1975/OR+si-ID1}

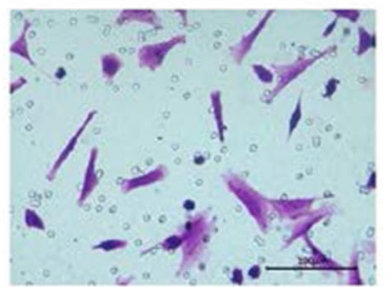

H1975/OR+si-ID1+pcDNA3.0-ID1

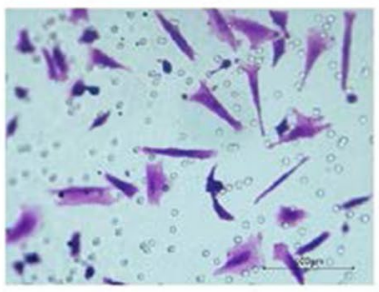

Fig. 4 Transwell assay of osimertinib-sensitive and resistant cells. Results showed that cell invasion was lowest in H1975 cells. Down-regulating ID1 could decrease cell invasion in H1975/OR + si-ID1 cells, while overexpression of ID1 could further enhance cell invasion in H1975/ OR + pcDNA3.0-ID1 cells. The values represent mean \pm SD from 3 independent experiments. ${ }^{*} \mathrm{P}<0.05$ versus H1975/OR

Impact of ID1 on apoptosis and the cell cycle of osimertinib-sensitive and resistant cells In our study, we found that alteration of ID1 expression in H1975/OR cells could change apoptosis induced by osimertinib. The apoptosis rate in H1975/OR cells was significantly decreased compared with that 
in osimertinib-sensitive cells $(13.63 \pm 1.72 \%$ versus $24.7 \pm 0.78 \%)$. In $\mathrm{H} 1975 / \mathrm{OR}+$ si-ID1 cells, the apoptosis rate was increased compared to H1975/OR cells $(22.07 \pm 1.03 \%$ versus $13.63 \pm 1.72 \%)$, whereas in ID1 overexpressed H1975/OR + pcDNA3.0-ID1 cells, the apoptosis rate was further decreased $(13.63 \pm 1.72 \%$ versus $8.73 \pm 1.94 \%$ ) (Fig. 5). This result was in accordance with that of the sensitive cells, which indicated that by inhibiting the expression of ID1, the drug resistance of osimertinib could be reversed in H1975/OR cells. To confirm this phenomenon, we checked the result in the negative control group. The rate of cell apoptosis did not change significantly compared to that in H1975/OR cells.

We conducted further experiment to investigate the impact of ID1 on the cell cycle of osimertinib-sensitive and resistant cells. The ratio of different cell cycle

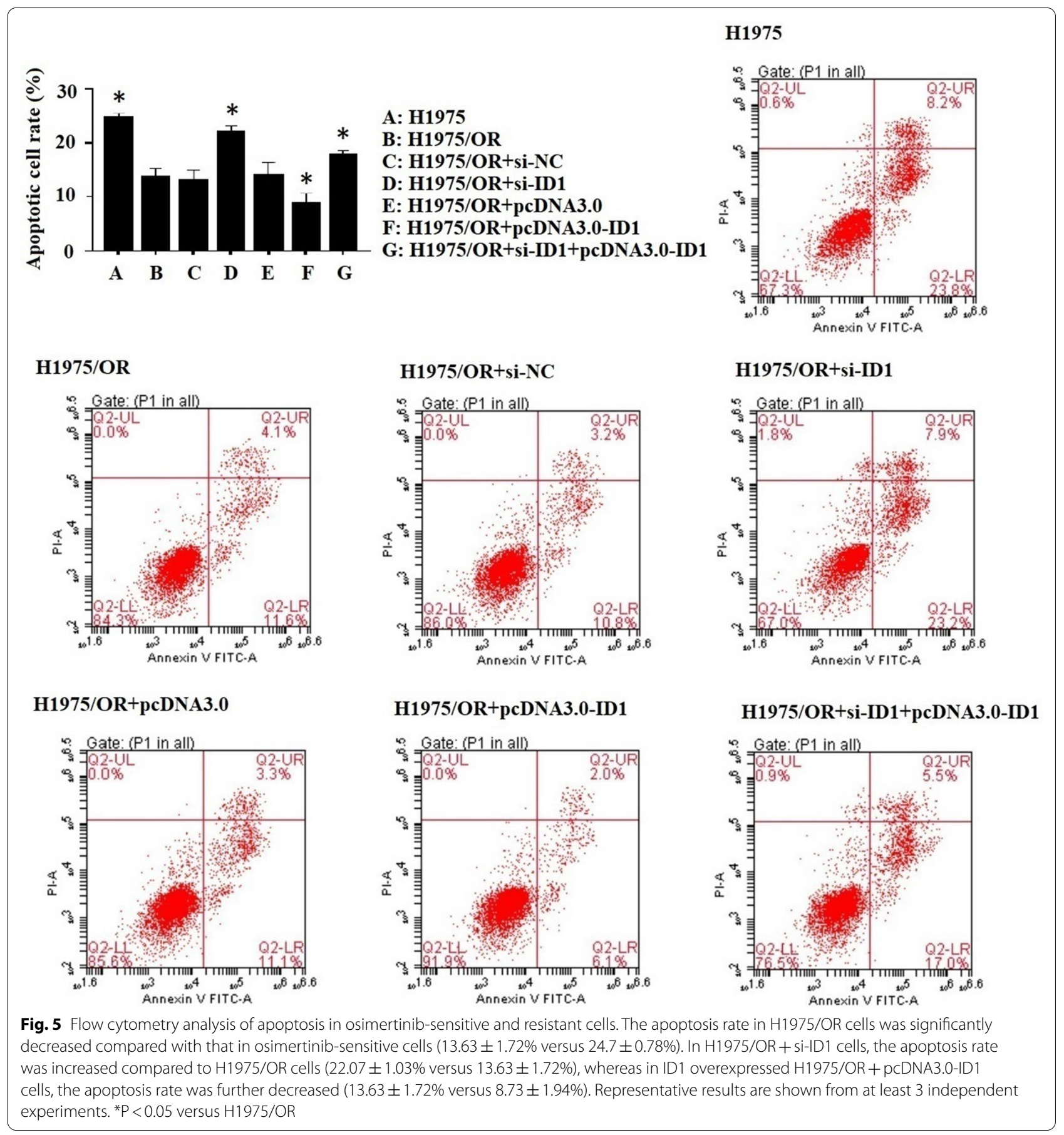


phases in H1975/OR cells is as following: the G1/G0 phase $(56.15 \pm 0.77 \%)$, the $S$ phase $(25.59 \pm 1.47 \%)$, the G2/M phase $(18.26 \pm 2.22 \%)$. As compared to H1975/ OR cells, cells were arrested in the G1/G0 phase in H1975 cells $(56.15 \pm 0.77 \%$ versus $68.29 \pm 1.24 \%$ ). By down-regulating ID1, this result was reversed in $\mathrm{H} 1975 / \mathrm{OR}+\mathrm{si}-\mathrm{ID} 1$ cells $(56.15 \pm 0.77 \%$ versus $63.05 \pm 0.82 \%$ ), while in ID1 overexpressed H1975/ OR + pcDNA3.0-ID1 cells, the effect on cell cycle was further aggravated $(56.15 \pm 0.77 \%$ versus $49.96 \pm 0.44 \%)$ (Fig. 6). Moreover, our results indicated that ID1 overexpression significantly reduced the number of cells in G1/G0 phase compared with the pc-control group. By contrast, ID1 knockdown increased the number of cells in G1/G0 phase compared with the si-control group, which was similar to that in the osimertinib-sensitive H1975 cells.

\section{Discussion}

Currently, first-line treatment for patients with EGFR mutated advanced NSCLC includes single targeted agents such as osimertinib, gefitinib and erlotinib. For lung cancer patients with EGFR T790M mutation, osimertinib as first-line treatment or as subsequent treatment after initial targeted therapy should be given priority [7]. However, osimertinib may still face the problem of drug resistance after a period of therapeutic time. The mechanism of osimertinib resistance is very complex. The most important mechanism is the C797S mutation of the EGFR gene [20]. The C797S mutation is

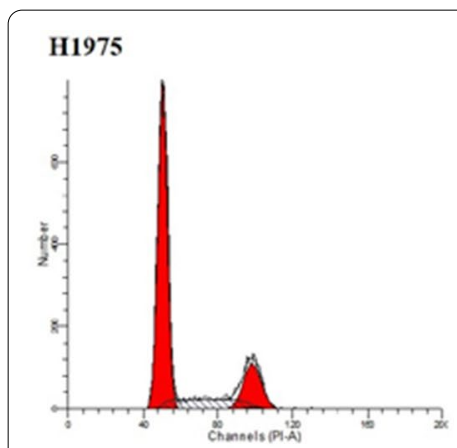

H1975/OR

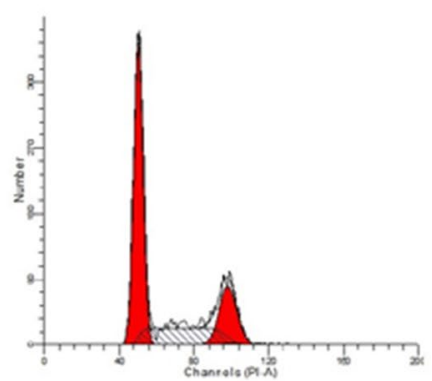

H1975/OR+pcDNA3.0

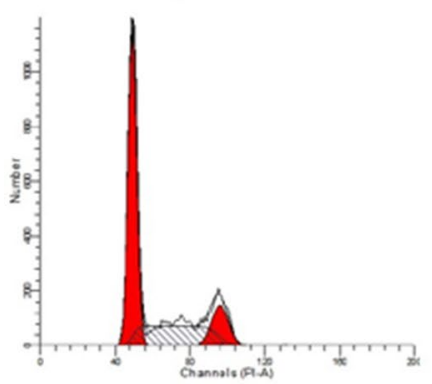

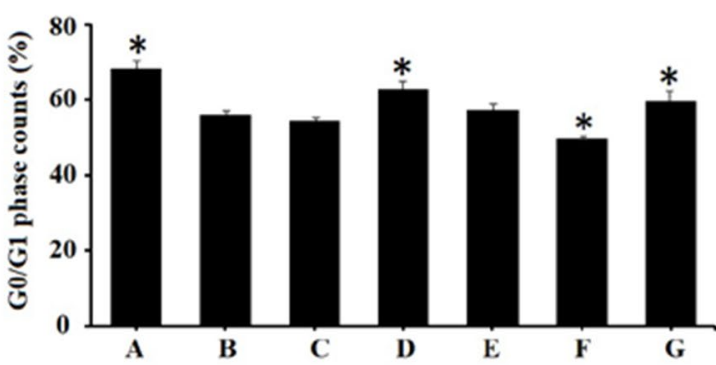

A: $\mathrm{H1975}$

B: $\mathrm{H} 1975 / \mathrm{OR}$

C: $\mathrm{H} 1975 / \mathrm{OR}+\mathrm{si}-\mathrm{NC}$

D: $\mathrm{H} 1975 / \mathrm{OR}+$ si-ID1

E: H1975/OR+pcDNA3.0

F: H1975/OR+pcDNA3.0-ID1

G: H1975/OR+si-ID1+pcDNA3.0-ID1

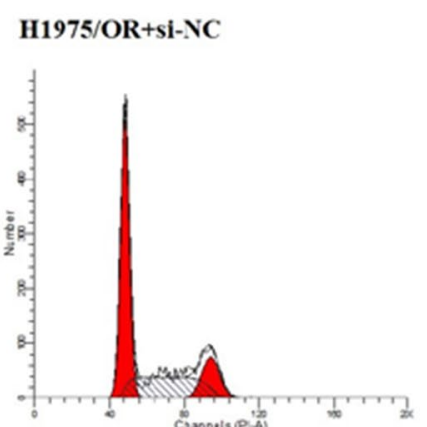

H1975/OR+pcDNA3.0-ID1

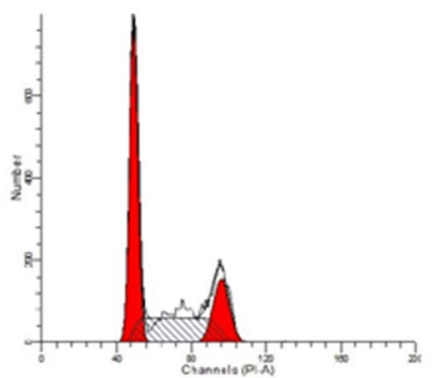

H1975/OR+si-ID1

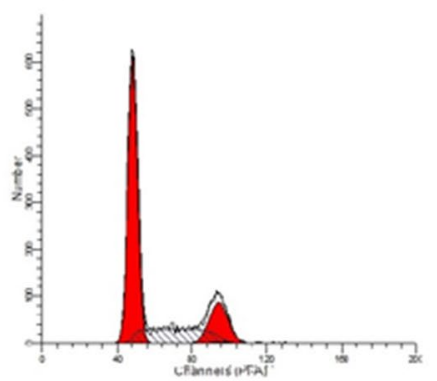

H1975/OR+si-ID1+pcDNA3.0-ID1

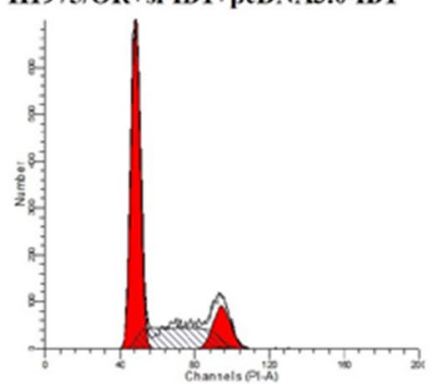

Fig. 6 Flow cytometry analysis of the cell cycle of osimertinib-sensitive and resistant cells. Compared with H1975/OR cells, cells were arrested in the G1/G0 phase in $\mathrm{H} 1975$ cells ( $56.15 \pm 0.77 \%$ versus $68.29 \pm 1.24 \%$ ). By down-regulating ID1, this result was reversed in H1975/OR + si-ID1 cells $(56.15 \pm 0.77 \%$ versus $63.05 \pm 0.82 \%)$, while in ID1 overexpressed H1975/OR + pcDNA3.0-ID1 cells, the effect on cell cycle was further aggravated $(56.15 \pm 0.77 \%$ versus $49.96 \pm 0.44 \%)$. Representative results are shown from at least 3 independent experiments. ${ }^{*} P<0.05$ versus H1975/OR 
located in the EGFR tyrosine kinase region, which can inhibit the effect of osimertinib and mediate the drug resistance of lung cancer cells. For the C797S mutation, EAI045 has been developed to overcome osimertinib resistance [21].

For patients without the C797S mutation, it has been reported that HER2 or c-Met amplification pathways are involved in osimertinib resistance [22]. Abnormal activation of HER2 can activate downstream pathways such as PI3K/Akt and MEK/MAPK through the formation of homologous or heterologous dimers, which leads to abnormal proliferation of tumor cells and the production of osimertinib resistance. Additionally, c-Met amplification bypasses EGFR activation of the downstream PI3K/ Akt-mediated signaling pathway, leading to osimertinib resistance. The activation of Ras, a downstream signaling pathway, can also lead to osimertinib resistance [23]. In addition to these processes, there are still many unknown mechanisms of osimertinib resistance, especially in the front-line settings. Therefore, investigating the resistance mechanism of osimertinib and overcoming the resistance problem of the third generation EGFR-TKIs have become one of the research focuses for NSCLC.

Till date, several resistance mechanisms have been described in previous studies such as the FLAURA and AURA3 trials. However, it was still unclear whether other mechanisms of acquired resistance to osimertinib existed when used as front line treatment. As were shown in previous studies, EMT can be resistant to traditional anticancer drugs and EGFR-TKIs. EMT can promote the growth, migration and metastasis of tumor cells. It has been found that EMT tumor cells have the ability for selfrenewal, unlimited proliferation and anti-apoptosis, and highly express CD133, CD44+ and ABCG2. These characteristics are very similar to those of cancer stem cells, which may be the potential mechanism of osimertinib resistance [24].

In our study, we found that in osimertinib-resistant NSCLC cells, the expression level of the EMT-related protein E-cadherin was lower than that in sensitive cells, while the expression level of ID1 and vimentin were higher than those in sensitive cells. Furthermore, ID1 expression level was closely related to E-cadherin and vimentin both in both osimertinib-sensitive and resistant cells. Alteration of ID1 expression in H1975/ OR cells could change the expression of E-cadherin. Downregulating ID1 expression in H1975/OR cells could inhibit cell proliferation, reduce cell invasion, promote cell apoptosis and arrested the cell cycle in the G1/G0 stage phase. Our study indicated that ID1 could induce EMT in EGFR T790M-positive NSCLC cells, which mediates drug resistance to osimertinib. These results may compensate the pre-existing osimertinib resistance mechanisms such as C797S mutation, c-Met amplification and downstream pathways activation.

In previous studies, ID1 was shown to participate in liver metastasis of lung cancer cells through EMT and knockout of ID1 may lead to decreased expression of vimentin, TGF- $\beta$ and Snail [25]. Down-regulation of ID1 expression can not only inhibit EMT formation, but can also induce tumor apoptosis. It has been reported that ID1 knockout in ovarian cancer cells significantly inhibited the growth and invasion of tumor cells and promoted the apoptosis of tumor cells [26]. In colon cancer HCT116 cells, ID1 inhibited apoptosis induced by chemotherapy drugs and ultraviolet light [27]. In small cell lung cancer, it has been reported that the high expression of ID1 can significantly inhibit the apoptosis of tumor cells [28]. Our study is therefore consistent with the results of these former studies.

In head and neck cancer cells, Snail-induced EMT enabled cancer cells to maintain their tumor stem celllike properties, thus increasing resistance to chemotherapy and invasiveness [29]. When tumor cells produce EMT, they often secrete more cytokines such as CXCL9 and CXCL10, which can inhibit the function of NK cells, thus promoting tumor immune escape [30]. Our study found that in EGFR T790M-positive NSCLC cells, increased ID1 expression could mediate EMT induction, inhibit cell apoptosis and promote cell proliferation. Down-regulation of ID1 expression could arrest the cell cycle in the G1/G0 phase. After ID1 expression level changes, does the proportion of tumor stem cells change? At present, there is no report that ID1-mediated EMT promotes the formation of tumor stem cells to regulate the immune escape of EGFR T790M-positive lung cancer. We will therefore conduct further studies to investigated these mechanisms.

However, our study has several limitations. Firstly, the role of ID1 in osimertinib resistance was only explored in H1975 and H1975/OR cells. It is difficult to confirm whether ID1 is definitely related to the drug resistance of osimertinib in other cell lines. Therefore, further studies are warranted to include more NSCLC cell lines harboring EGFR T790M mutation. Secondly, due to a shortage in time and funding, several experiments were not investigated in the current study, such as the correlation between ID1, EMT downstream signaling pathways and immune escape after osimertinib resistance. Furthermore, our study was only conducted in vitro, and in vivo experiments in models such as the nude mouse xenograft model were not performed. Hence, the role of ID1 needs to be carefully verified in animal experiments. Lastly, our study did not involved in human samples as compared to other similar researches. As a consequence, further exploration 
of ID1 in EGFR T790M-positive lung cancer cells is required.

\section{Conclusions}

In conclusion, our study revealed the mechanism of ID1 mediated resistance to osimertinib in EGFR T790M-positive NSCLC through EMT, which may provide new ideas and methods for the clinical treatment of EGFR mutated NSCLC after osimertinib resistance. Further studies are needed to elucidate the underlying mechanism of osimertinib resistance in first-line targeted therapy.

\section{Abbreviations}

NSCLC: Non-small cell lung cancer; EGFR: Epidermal growth factor receptor; TKIs: Tyrosine kinase inhibitors; EMT: Epithelial-mesenchymal transition; PFS: Progression-free survival; bHLH: Basic helix-loop-helix; CCK-8: Cell Counting Kit-8 assay; SDS-PAGE: Sodium dodecyl sulfate polyacrylamide gel electrophoresis; PGK: Phosphoglycerate kinase; RT-PCR: Reverse transcription-PCR.

\section{Supplementary Information}

The online version contains supplementary material available at https://doi. org/10.1186/s12890-021-01540-4.

Additional file 1. Western blot of ID1 expression.

Additional file 2. Western blot of EMT-related proteins.

Additional file 3. Western blot of different ID1 expression and EMTrelated proteins.

\section{Acknowledgements}

The authors would like to thank colleagues at Affiliated Dongguan People's Hospital, Southern Medical University and Gaozhou People's Hospital for their kind technical help, psychological support, theoretical guidance and writing assistance during the present study.

\section{Authors' contributions}

KJL, XWC and LGW conducted the experiment and participated in drafting and revising the manuscript. SYC did the statistical work. NXF, LMC and JJ designed and supervised the process of whole study. All authors read and approved the final manuscript and agreed to be accountable for all aspects of the work presented in the manuscript.

\section{Funding}

This study was funded by Research Development Project of Dongguan People's Hospital (Grant No. K201922).

\section{Availability of data and materials}

The datasets used and analyzed during the current study are available from the corresponding author on reasonable request.

\section{Declarations}

Ethics approval and consent to participate

Not applicable.

\section{Consent for publication}

Not applicable.

\section{Competing interests}

The authors declare that they have no competing interests.

\section{Author details}

${ }^{1}$ Dongguan Institute of Clinical Cancer Research, Affiliated Dongguan People's Hospital, Southern Medical University, Dongguan, China. ${ }^{2}$ Department of Pathology, Gaozhou People's Hospital, Maoming, China. ${ }^{3}$ Dongguan Institute of Respiratory and Critical Care Medicine, Affiliated Dongguan People's Hospital, Southern Medical University, Dongguan, China.

Received: 6 December 2020 Accepted: 11 May 2021

Published online: 15 May 2021

\section{References}

1. Siegel RL, Miller KD, Jemal A. Cancer statistics, 2020. CA Cancer J Clin. 2020;70(1):7-30.

2. Liu K, Jiang G, Zhang A, Li Z, Jia J. Icotinib is as efficacious as gefitinib for brain metastasis of EGFR mutated non-small-cell lung cancer. BMC Cancer. 2020;20(1):76.

3. Pérez-Soler R, Chachoua A, Hammond LA, Rowinsky EK, Huberman $M, \operatorname{Karp} D$, et al. Determinants of tumor response and survival with erlotinib in patients with non-small-cell lung cancer. J Clin Oncol. 2004;22(16):3238-47.

4. Kris MG, Natale RB, Herbst RS, Lynch TJ Jr, Prager D, Belani CP, et al. Efficacy of gefitinib, an inhibitor of the epidermal growth factor receptor tyrosine kinase, in symptomatic patients with NSCLC: a randomized trial. JAMA. 2003;290(16):2149-58.

5. Huang L, Fu L. Mechanisms of resistance to EGFR tyrosine kinase inhibitors. Acta Pharm Sin B. 2015;5(5):390-401.

6. Goss G, Tsai CM, Shepherd FA, Bazhenova L, Lee JS, Chang GC, et al. Osimertinib for pretreated EGFR Thr790Met-positive advanced non-smallcell lung cancer (AURA2): a multicentre, open-label, single-arm, phase 2 study. Lancet Oncol. 2016;17(12):1643-52.

7. MokTS, Wu YL, Ahn MJ, Garassino MC, Kim HR, Ramalingam SS, et al. Osimertinib or platinum-pemetrexed in EGFR T790M-positive lung cancer. N Engl J Med. 2017;376(7):629-40.

8. Gao S, Sun Y, Zhang X, Hu L, Liu Y, Chua CY, et al. IGFBP2 activates the NF-kB pathway to drive epithelial-mesenchymal transition and invasive character in pancreatic ductal adenocarcinoma. Cancer Res. 2016;76(22):6543-54.

9. Kim AY, Kwak JH, Je NK, Lee YH, Jung YS. Epithelial-mesenchymal transition is associated with acquired resistance to 5-Fluorocuracil in HT-29 colon cancer cells. Toxicol Res. 2015;31(2):151-6.

10. Liang YK, Zeng D, Xiao YS, Wu Y, Ouyang YX, Chen M, et al. MCAM/CD146 promotes tamoxifen resistance in breast cancer cells through induction of epithelial-mesenchymal transition, decreased ERa expression and AKT activation. Cancer Lett. 2017;386:65-76.

11. Song J, Li Y. miR-25-3p reverses epithelial-mesenchymal transition via targeting Sema4C in cisplatin-resistance cervical cancer cells. Cancer Sci. 2017;108(1):23-31.

12. Kurimoto R, Iwasawa S, Ebata T, Ishiwata T, Sekine I, Tada Y, et al. Drug resistance originating from a TGF- $\beta / F G F-2$-driven epithelial-to-mesenchymal transition and its reversion in human lung adenocarcinoma cell lines harboring an EGFR mutation. Int J Oncol. 2016;48(5):1825-36.

13. Rastogi I, Rajanna S, Webb A, Chhabra G, Foster B, Webb B, et al. Mechanism of c-Met and EGFR tyrosine kinase inhibitor resistance through epithelial mesenchymal transition in non-small cell lung cancer. Biochem Biophys Res Commun. 2016;477(4):937-44.

14. Bai XY, Zhang XC, Yang SQ, An SJ, Chen ZH, Su J, et al. Blockade of hedgehog signaling synergistically increases sensitivity to epidermal growth factor receptor tyrosine kinase inhibitors in non-small-cell lung cancer cell lines. PLoS ONE. 2016;11(3):e0149370.

15. Tong D, Liu Q, Liu G, Xu J, Lan W, Jiang Y, et al. Metformin inhibits castration-induced EMT in prostate cancer by repressing COX2/PGE2/ STAT3 axis. Cancer Lett. 2016;389:23-32.

16. Li B, Xu WW, Guan XY, Qin YR, Law S, Lee NP, et al. Competitive binding between Id1 and E2F1 to Cdc20 regulates E2F1 degradation and thymidylate synthase expression to promote esophageal cancer chemoresistance. Clin Cancer Res. 2016;22:1243-55.

17. Li B, Tsao SW, Li YY, Wang X, Ling MT, Wong YC, et al. Id-1 promotes tumorigenicity and metastasis of human esophageal cancer cells through activation of PI3K/AKT signaling pathway. Int J Cancer. 2009;125(11):2576-85. 
18. Baik IH, Jo GH, Seo D, Ko MJ, Cho CH, Lee MG, et al. Knockdown of RPL9 expression inhibits colorectal carcinoma growth via the inactivation of Id-1/NF-kB signaling axis. Int J Oncol. 2016;49(5):1953-62.

19. Bao Y, Zhao Y, Chen B, Luo J, Deng Q, Sun H, et al. Relationship between ID1 and EGFR-TKI resistance in non-small cell lung cancer. Zhongguo Fe Ai Za Zhi. 2016;19(12):864-70

20. Thress KS, Paweletz CP, Felip E, Cho BC, Stetson D, Dougherty B, et al. Acquired EGFR C797S mutation mediates resistance to AZD9291 in nonsmall cell lung cancer harboring EGFR T790M. Nat Med. 2015:21(6):560-2.

21. Jia Y, Yun CH, Park E, Ercan D, Manuia M, Juarez J, et al. Overcoming EGFR( T790M) and EGFR(C797S) resistance with mutant-selective allosteric inhibitors. Nature. 2016;534(7605):129-32.

22. Planchard D, Loriot Y, Andre F, Gobert A, Auger N, Lacroix L, et al. EGFRindependent mechanisms of acquired resistance to AZD9291 in EGFR T790M-positive NSCLC patients. Ann Oncol. 2015;26(10):2073-8.

23. Eberlein CA, Stetson D, Markovets AA, Al-Kadhimi KJ, Lai Z, Fisher PR, et al. Acquired resistance to the mutant-selective EGFR inhibitor AZD9291 is associated with increased dependence on RAS signaling in preclinical models. Cancer Res. 2015;75(12):2489-500.

24. Bhuvanesh D, Vivek M, Nicholas MT, Jenny CC. Epithelial-mesenchymal transition, cancer stem cells and treatment resistance. Breast Cancer Res. 2012;14(1):202-9.

25. Castañón E, Soltermann A, López I, Román M, Ecay M, Collantes $M$, et al. The inhibitor of differentiation-1 (Id1) enables lung cancer liver colonization through activation of an EMT program in tumor cells and establishment of the pre-metastatic niche. Cancer Lett. 2017;402:43-51.

26. Sun WZ, Li MH, Chu M, Wei LL, Bi MY, HeY, et al. Id1 knockdown induces the apoptosis and inhibits the proliferation and invasion of ovarian cancer cells. Eur Rev Med Pharmacol Sci. 2016;20(13):2812-8.

27. Zhao Y, Zhang W, Luo A, Wang X, Liu Z. ID1 suppress the apoptosis of HCT116 cells induced by chemotherapeutic drugs and ultraviolet radiation. Zhonghua Zhong Liu Za Zhi. 2016;38(1):4-10.

28. Chen D, Forootan SS, Gosney JR, Forootan FS, Ke Y. Increased expression of Id 1 and Id 3 promotes tumorigenicity by enhancing angiogenesis and suppressing apoptosis in small cell lung cancer. Genes Cancer. 2014;5(5-6):212-25.

29. Ota I, Masui T, Kurihara M, Yook JI, Mikami S, Kimura T, et al. Snail-induced EMT promotes cancer stem cell-like properties in head and neck cancer cells. Oncol Rep. 2016;35(1):261-6.

30. Poggi A, Giuliani M. Mesenchymal stromal cells can regulate the immune response in the tumor microenvironment. Vaccines (Basel). 2016;4(4):41.

\section{Publisher's Note}

Springer Nature remains neutral with regard to jurisdictional claims in published maps and institutional affiliations.
Ready to submit your research? Choose BMC and benefit from:

- fast, convenient online submission

- thorough peer review by experienced researchers in your field

- rapid publication on acceptance

- support for research data, including large and complex data types

- gold Open Access which fosters wider collaboration and increased citations

- maximum visibility for your research: over 100M website views per year

At BMC, research is always in progress.

Learn more biomedcentral.com/submissions 